Cahiers $d u$ MONDE RUSSE

\section{Cahiers du monde russe}

Russie - Empire russe - Union soviétique et États indépendants

42/2-4 | 2001

La police politique en Union soviétique, 1918-1953

\title{
Les transformations du personnel des organes de sécurité soviétiques, 1922-1953
}

\section{Nikita PETROV}

\section{OpenEdition}

Journals

Édition électronique

URL : https://journals.openedition.org/monderusse/8460

DOI : $10.4000 /$ monderusse. 8460

ISSN : $1777-5388$

\section{Éditeur}

Éditions de l'EHESS

\section{Édition imprimée}

Date de publication : 1 avril 2001

Pagination : 375-396

ISBN : 2-7132-1398-3

ISSN : $1252-6576$

Référence électronique

Nikita PETROV, «Les transformations du personnel des organes de sécurité soviétiques,

1922-1953 », Cahiers du monde russe [En ligne], 42/2-4 | 2001, mis en ligne le 01 janvier 2007, consulté

le 02 septembre 2022. URL : http://journals.openedition.org/monderusse/8460 ; DOI : https://doi.org/ 10.4000/monderusse. 8460 
chercher : repérer : avancer

Cet article est disponible en ligne à l'adresse :

http://www.cairn.info/article.php?ID REVUE=CMR\&ID NUMPUBLIE=CMR 422\&ID ARTICLE=CMR 4220375

Les transformations du personnel des organes de sécurité soviétiques, 19221953

par Nikita PETROV

| Editions de l'EHESS | Cahiers du monde russe

2001/2-3-4 - Vol 42

ISSN 1252-6576 | ISBN 2713213983 | pages 375 à 396

Pour citer cet article :

- Petrov N., Les transformations du personnel des organes de sécurité soviétiques, 1922-1953, Cahiers du monde russe 2001/ 2-3-4, Vol 42, p. 375-396.

Distribution électronique Cairn pour les Editions de l'EHESS.

(C) Editions de l'EHESS. Tous droits réservés pour tous pays.

La reproduction ou représentation de cet article, notamment par photocopie, n'est autorisée que dans les limites des conditions générales d'utilisation du site ou, le cas échéant, des conditions générales de la licence souscrite par votre établissement. Toute autre reproduction ou représentation, en tout ou partie, sous quelque forme et de quelque manière que ce soit, est interdite sauf accord préalable et écrit de l'éditeur, en dehors des cas prévus par la législation en vigueur en France. Il est précisé que son stockage dans une base de données est également interdit. 


\title{
LES TRANSFORMATIONS DU PERSONNEL DES ORGANES DE SÉCURITÉ SOVIÉTIQUES, 1922-1953
}

\begin{abstract}
Au cours de ces dernières années, plusieurs travaux ont été consacrés à la formation et à la hiérarchisation des couches dirigeantes de l'appareil du parti et de l'État, à l'analyse du personnel et aux modifications qualitatives de l'élite soviétique au pouvoir en 1937-1938, consécutives aux purges staliniennes conduites parmi les cadres du parti, des soviets, de l'armée et des sphères économiques ${ }^{1}$. Aucun organe ne s'est trouvé épargné, pas même le commissariat du peuple aux Affaires intérieures (NKVD) au sein duquel plusieurs générations de fonctionnaires tchékistes ont été remplacées entre 1934 et 1941. La modification des effectifs du NKVD avant et après la «Grande Terreur » est si importante qu'il n'est pas exagéré de parler de véritable révolution. Afin de mieux comprendre les changements intervenus au cours de cette décennie, il convient au préalable de rappeler certains des paramètres les plus importants et les plus caractéristiques de la composition des cadres de la VČK-OGPU-NKVD-NKGB-MGB aux différents moments de la période étudiée et de mettre en évidence la logique des mouvements de cadres observés.
\end{abstract}

Au $1^{\text {er }}$ décembre 1922, le personnel de l'appareil central de la GPU comptait 2213 collaborateurs. Au cours de la période 1921-1923, on observe une réduction significative des effectifs globaux de la VČK-GPU à travers le pays. En effet, à la fin de l'année 1921, on recense 90000 collaborateurs rémunérés au budget (glasnye sotrudniki) à la VČK. Lors de la création de la GPU, en février 1922, leur

\footnotetext{
1. M. S. Voslenskij, Nomenklatura. Gospodstvujuščij klass Sovetskogo Sojuza (La Nomenklatura. La classe dirigeante de l'Union Soviétique), M., 1991 ; L. E. Bonjuškina, « Opyt izučenija stanovlenija professional'noj sovetskoj elity (SNK 1937-1941 gg.) » (Essai d'étude de la mise en place d'une élite professionnelle soviétique, le SNK 1937-1941), Mir Rossii, 3-4, 1995, pp. 108-130 ; O. V. Naumov, S. G. Filippov, « Rukovodjaččij patijnyj rabotnik v 1924 i 1937 gg. Popytka sravnitel'nogo analiza » (L'ouvrier du parti aux commandes des années 1924 à 1937. Essai d'analyse comparée), Social'naja istorija. Ežegodnik, 1998, pp. 123-136.
} 
nombre se limite à 60000 et se réduit encore au cours de l'année. Finalement, au $1^{\text {er }}$ novembre 1923, il ne restait plus que 33152 personnes dans les circuits de la GPU. Une évolution similaire a affecté les collaborateurs rémunérés sur les « fonds secrets » (sekretnye ou neglasnye sotrudniki): début 1921, ils étaient 60000 et moitié moins un an plus tard. Au 1 ${ }^{\text {er }}$ novembre 1923 le personnel «secret» ne comptait plus que 12900 agents. Une tendance comparable peut être observée au sein de l'armée. En octobre 1922, le chiffre total des troupes de l'intérieur (vnytrennie vojska), des gardes-frontières (pogračnye vojska) et des troupes d'escorte (konvojnye vojska) s'élevait à 117000 hommes, alors qu'un an plus tard, en novembre 1923, il n'était plus que de 74800 hommes, dont 24800 rattachés aux forces armées de l'intérieur, 32977 aux gardes-frontières et 17000 aux troupes d'escorte 2 .

Au cours des années qui ont suivi, le nombre total des employés de l'OGPU a continué de décroître. Au milieu de l'année 1930, il n'était que de 22180 pour l'ensemble du territoire (non compris les troupes de l'OGPU et les gardes-frontières) $)^{3}$. Puis, à la suite de la réforme administrativo-territoriale de juillet-août 1930 et conformément à la réorganisation des structures territoriales de l'OGPU, le personnel des organes de sécurité totalisait, au 1 ${ }^{\mathrm{er}}$ octobre 1930, 25288 agents $^{4}$. Ce chiffre intégrait toutes les catégories d'employés de l'OGPU y compris le personnel du secrétariat et le personnel de service. Si l'on compte à part le personnel « opérationnel » (operativnyj sostav), selon les données statistiques fournies dans les rapports de la section des cadres de l'OGPU-NKVD, la composition des organes de sécurité était la suivante ${ }^{5}$ :

\begin{tabular}{|l|l|}
\hline au 01.01 .1933 & 20898 personnes \\
\hline au 01.01 .1934 & 25022 personnes \\
\hline au 01.01 .1935 & 25573 personnes \\
\hline au 01.07 .1935 & 23188 personnes \\
\hline
\end{tabular}

En outre, le personnel du secrétariat et le personnel de service des organes de sécurité (UGB-GUGB: (Glavnoe) Upravlenie Gosudarstvennoj Bezopasnosti - Direction de la sécurité d'État) comptait, au $1^{\text {er juillet 1935, } 1519 \text { personnes }}{ }^{6}$.

Les variations quantitatives du personnel opérationnel de l'OGPU-NKVD au début des années 30 s'expliquent par les restructurations opérées. Ainsi, fin 1932,

2. A. Kokurin, N. Petrov, « GPU-OGPU (1922-1928) », Svobodnaja mysl’, 7, 1998, p. 115.

3. Cf., pour plus de détails, N. V. Petrov, K. V. Skorkin, Kto rukovodil NKVD, 1934-1941: Spravočnik / Obščestvo «Memorial », RGASPI, GARF (Qui dirigeait le NKVD, 1934-1941: Annuaire / Association « Mémorial », RGASPI, GARF), sous la dir. de N. G. Ohotin et A. B. Roginskij, Moscou, Zven’ja, 1999, p. 35.

4. Ibid.

5. GARF, f. 9401, op. 8, d. 40,11. 1-2.

6. Ibid. 
des postes de directeur-adjoint des sections politiques des stations de machines et de tracteurs ainsi que des sovkhozes furent créés dans le cadre de l'OGPU. On nomma exclusivement à ces postes des collaborateurs de l'OGPU auxquels furent conférés exactement les mêmes mandats qu'aux directeurs des sections de districts (rajonnye otdely) de la GPU. En conséquence, les effectifs du personnel opérationnel de l'OGPU-NKVD pour l'ensemble du pays se sont accrus en 1933 de 4124 personnes et, en 1934 , de 551 personnes ${ }^{7}$. Durant la première moitié de l'année 1935, ces postes furent supprimés au profit d'environ 800 nouvelles sections de districts et de nouveaux départements du NKVD, ce qui a entraîné une réduction de 2385 personnes du nombre des agents opérationnels du NKVD ${ }^{8}$.

Il convient de revenir plus particulièrement sur la composition nationale des organes de l'OGPU durant les années 20, question qui a déjà fait l'objet de différents travaux, quoique peu nombreux ${ }^{9}$. Dans ces études, l'accent a été mis sur la représentation importante et disproportionnée des Lettons, des Juifs et des Polonais dans les organes répressifs soviétiques. Ainsi, d'après les données publiées par L. Ju. Kričevskij, dans les effectifs de la cellule du parti de l'appareil central de l'OGPU qui comptait 980 personnes en janvier 1925, les Russes représentaient $66,5 \%$, du total, les Lettons $-16,7 \%$, les Juifs $-7,6 \%$, et les Polonais $-6,1 \% 10$. S'agissant de l'ensemble des collaborateurs de l'appareil central de l'OGPU, la représentation en fonction de la nationalité (au 1er mai 1924) était la suivante: sur un total de 2402 employés, les Russes représentaient 69,5\% de l'ensemble, les Lettons $-8,6 \%$, les Juifs $-8,5 \%$ et les Polonais $3,7 \%{ }^{11}$.

L'origine sociale des agents des services de sécurité n'était pas non plus représentative des différents groupes sociaux existant en URSS au milieu des années 30. En effet, la catégorie de tchékistes «d'origine sociale étrangère » (čuždogo proishoždenija) était sur-représentée. En revanche, les employés issus de familles paysannes étaient très peu nombreux. D'après le bilan établi au $1^{\mathrm{er}}$ juillet 1935 , sur un total de 23188 collaborateurs des services de sécurité, 44,8\% étaient issus de la classe ouvrière $; 13,7 \%$ de la paysannerie, et $41,5 \%$ de la catégorie des employés ${ }^{12}$. Certes, le phénomène peut facilement être expliqué par le fait que, jusqu'en 1917, les enfants de parents aisés pouvaient recevoir une assez bonne formation. Autrement dit, ce sont les fils d'anciens fonctionnaires, de marchands et de petits entrepreneurs qui constituaient la partie la plus lettrée du personnel opérationnel. Cepen-

\section{Ibid.}

8. Ibid.

9. Cf. L. Ju. Kričevskij, « Evrei v apparate VČK-OGPU v 20-e gody » (Les Juifs dans l'appareil de la VČK-OGPU dans les années 20), in O. Budnickij, ed., Evrei i russkaja revoljucija. Materialy $i$ issledovanija (Les Juifs et la révolution russe. Matériaux et recherches), MoscouJérusalem, 1999, pp. 320-350 ; O. I. Kapčinskij, Nacional’nyj sostav central'nogo apparata OGPU v 1920-e gody (La composition nationale de l'appareil central de l'OGPU dans les années 1920, http//www.fsb.history/read/1999/kapchinsky.html

10. L. Ju Kričevskij, art. cit., p. 335.

11. Ibid., p. 334.

12. GARF, f. 9401 , op. 8 , d. $40,1.10$. 
dant, le niveau général de formation de l'encadrement opérationnel laissait à

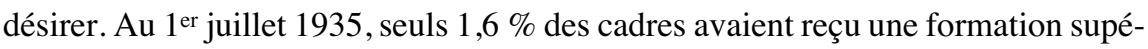
rieure, $23,8 \%$ une formation secondaire et la majorité, soit 74,6\%, ne possédaient qu'une formation élémentaire ${ }^{13}$.

Des contradictions semblables se manifestaient, avec plus de relief encore, à l'échelle des cadres supérieurs de la direction de l'OGPU-NKVD, comme nous le verrons par la suite en décrivant les changements observés dans les caractéristiques socio-politiques de l'oligarchie dirigeante du NKVD avant, pendant et après la « Grande Terreur» de 1937-193814.

Les Tableaux 1 à 9 font état de la dynamique des changements pour les indicateurs suivants: âge, origine sociale, composition nationale, niveau de formation, durée des fonctions à la Tchéka, ancienneté dans le parti (RSDRP(b)-VKP(b)), appartenance dans le passé à d'autres partis, données concernant les arrestations et les jugements durant la période 1937-194115.

Dans tous ces tableaux, les informations sont données en chiffres absolus et, entre parenthèses, en pourcentage du personnel total pour la période considérée.

\section{Ibid.,1. 16 .}

14. L'étude actuelle est fondée sur des informations biographiques et sur les effectifs déclarés de tchékistes, cités in N. V. Petrov, K. V. Skorin, op. cit. Les quelques distinctions de données dans les tableaux fournies dans l'ouvrage et ici même s'expliquent par la précision des renseignements sur les répressions (dates d'arrestation et de jugement) des dirigeants tchékistes. Dans notre étude, sont pris en compte les commissaires du peuple aux Affaires intérieures d'URSS et leurs adjoints, les chefs des directions et des sections de l'appareil central du NKVD, les commissaires du peuple aux Affaires intérieures de toutes les républiques de l'Union et des républiques autonomes (à l'exception de la République autonome du Nakhitchévan), les chefs des directions régionales (UNKVD) dans les kraja et oblasti de RSFSR, d'Ukraine, de Biélorussie et du Kazakhstan. Nous n'avons pas pris en considération les dirigeants du NKVD des oblasti autonomes de RSFSR qui n'ont pas changé de statut administratif durant la période considérée, ni les dirigeants du NKVD des oblasti de Kirghizie, du Tadjikistan, du Turkménistan, et de l'Ouzbékistan. Nous avons tenu compte des dirigeants de l'UNKVD des oblasti autonomes de RSFSR ayant accédé au statut de républiques autonomes.

15. Le nombre total des dirigeants du NKVD a constamment augmenté, de juillet 1934 à février 1941, du fait de la formation de nouvelles unités administratives et territoriales, et aussi en raison des changements structurels de l'appareil central du NKVD, de la création de nouvelles directions et de nouvelles sections. Les effectifs du NKVD travaillant à l'échelon considéré ont presque doublé, passant de 96 à 182 personnes. On a pu observer un accroissement particulièrement élevé en 1938-1939, phénomène dû avant tout à l'élargissement de la structure de l'appareil central du NKVD. Le nombre de dirigeants de chaque séquence chronologique s'établit comme suit: au 10.07.34-96, au 1.10.36-110, au 1.03.37-111, au 1.07.37-113, au 1.01.38 - 128, au 1.09.38 - 150, au 1.07.39-153, au 1.01.40 - 172 et, enfin, au 26.02.41 - 182 personnes. Comme on l'a déjà indiqué précédemment, les chiffres en pourcentage fournis dans les Tableaux 1 à 7 prennent en compte les effectifs d'ensemble des dirigeants en fonction durant chaque séquence chronologique. En outre, il faut noter que le nombre assez élevé de données imprécises pour la période 1937-1939 s'explique essentiellement par l'existence d'un nombre important de postes vacants chez les dirigeants, ce qui se comprend aisément dans le contexte des arrestations et des purges opérées. 
Tableau 1. Nombre de dirigeants du NKVD en fonction de leur âge

\begin{tabular}{|l|l|l|l|l|l|l|l|l|l|}
\hline $\begin{array}{l}\text { Année } \\
\text { de naissance }\end{array}$ & 10.07 .34 & $\mathbf{1 . 1 0 . 3 6}$ & $\mathbf{1 . 0 3 . 3 7}$ & $\mathbf{1 . 0 7 . 3 7}$ & $\mathbf{1 . 0 1 . 3 8}$ & $\mathbf{1 . 0 9 . 3 8}$ & $\mathbf{1 . 0 7 . 3 9}$ & $\mathbf{1 . 0 1 . 4 0}$ & $\mathbf{2 6 . 0 2 . 4 1}$ \\
\hline 1895 et avant & $\begin{array}{l}54 \\
(56,2 \%)\end{array}$ & $\begin{array}{l}52 \\
(47,3 \%)\end{array}$ & $\begin{array}{l}49 \\
(44,1 \%)\end{array}$ & $\begin{array}{l}37 \\
(32,7 \%)\end{array}$ & $\begin{array}{l}23 \\
(17,9 \%)\end{array}$ & $\begin{array}{l}23 \\
(15,3 \%)\end{array}$ & $\begin{array}{l}6 \\
(3,9 \%)\end{array}$ & $\begin{array}{l}5 \\
(2,9 \%)\end{array}$ & $\begin{array}{l}9 \\
(4,9 \%)\end{array}$ \\
\hline $1896-1900$ & $\begin{array}{l}35 \\
(36,5 \%)\end{array}$ & $\begin{array}{l}45 \\
(40,9 \%)\end{array}$ & $\begin{array}{l}48 \\
(43,2 \%)\end{array}$ & $\begin{array}{l}50 \\
(44,2 \%)\end{array}$ & $\begin{array}{l}64 \\
(50 \%)\end{array}$ & $\begin{array}{l}64 \\
(42,6 \%)\end{array}$ & $\begin{array}{l}25 \\
(16,3 \%)\end{array}$ & $\begin{array}{l}25 \\
(14,5 \%)\end{array}$ & $\begin{array}{l}29 \\
(15,9 \%)\end{array}$ \\
\hline $1901-1905$ & $\begin{array}{l}6 \\
(6,2 \%)\end{array}$ & $\begin{array}{l}11 \\
(10 \%)\end{array}$ & $\begin{array}{l}12 \\
(10,8 \%)\end{array}$ & $\begin{array}{l}16 \\
(14,1 \%)\end{array}$ & $\begin{array}{l}30 \\
(23,4 \%)\end{array}$ & $\begin{array}{l}49 \\
(32,6 \%)\end{array}$ & $\begin{array}{l}64 \\
(41,8 \%)\end{array}$ & $\begin{array}{l}77 \\
(44,7 \%)\end{array}$ & $\begin{array}{l}84 \\
(46,1 \%)\end{array}$ \\
\hline $1906-1910$ & - & - & - & - & - & $\begin{array}{l}2 \\
(1,3 \%)\end{array}$ & $\begin{array}{l}43 \\
(28,1 \%)\end{array}$ & $\begin{array}{l}52 \\
(30,2 \%)\end{array}$ & $\begin{array}{l}53 \\
(29,1 \%)\end{array}$ \\
\hline 1911 et après & - & - & - & - & - & - & $\begin{array}{l}7 \\
(4,6 \%)\end{array}$ & $\begin{array}{l}7 \\
(4,1 \%)\end{array}$ & $\begin{array}{l}6 \\
(3,3 \%)\end{array}$ \\
\hline $\begin{array}{l}\text { données } \\
\text { manquantes }\end{array}$ & $\begin{array}{l}1 \\
(1 \%)\end{array}$ & $\begin{array}{l}2 \\
(1,8 \%)\end{array}$ & $\begin{array}{l}2 \\
(1,8 \%)\end{array}$ & $\begin{array}{l}10 \\
(8,8 \%)\end{array}$ & $\begin{array}{l}11 \\
(8,6 \%)\end{array}$ & $\begin{array}{l}12 \%) \\
(8 \%)\end{array}$ & $\begin{array}{l}8 \\
(5,2 \%)\end{array}$ & $\begin{array}{l}6 \\
(3,5 \%)\end{array}$ & $\begin{array}{l}1 \\
(0,5 \%)\end{array}$ \\
\hline
\end{tabular}

Ce premier tableau montre que, durant la période considérée, un rajeunissement significatif s'est opéré dans le noyau dirigeant du NKVD. Ainsi, en 1937, plus de la moitié des dirigeants avaient 40 ans et plus, tandis qu'en 1939-1940 l'écrasante majorité des cadres avaient entre 30 et 35 ans. Ce constat correspond tout à fait à la réduction du stage dans les « organes » qui a caractérisé les générations d'employés de la Tchéka appelés par Berija (cf. Tableau 5). D’autre part, le changement s'explique également par le fait qu'en décembre 1938 et janvier 1939, on a procédé à un « recrutement du parti » (partijnyj nabor). Des responsables du parti, des soviets et de l'économie ont été dirigés vers des postes de direction dans les circuits du NKVD sur ordre du Comité central.

Tableau 2. Nombre de dirigeants du NKVD selon l'origine sociale

\begin{tabular}{|l|l|l|l|l|l|l|l|l|l|}
\hline $\begin{array}{c}\text { Catégorie } \\
\text { sociale }\end{array}$ & $\mathbf{1 0 . 0 7 . 3 4}$ & $\mathbf{1 . 1 0 . 3 6}$ & $\mathbf{1 . 0 3 . 3 7}$ & $\mathbf{1 . 0 7 . 3 7}$ & $\mathbf{1 . 0 1 . 3 8}$ & $\mathbf{1 . 0 9 . 3 8}$ & $\mathbf{1 . 0 7 . 3 9}$ & $\mathbf{1 . 0 1 . 4 0}$ & $\mathbf{2 6 . 0 2 . 4 1}$ \\
\hline ouvriers & $\begin{array}{l}23 \\
(24 \%)\end{array}$ & $\begin{array}{l}23 \\
(20,9 \%)\end{array}$ & $\begin{array}{l}26 \\
(23,4 \%)\end{array}$ & $\begin{array}{l}27 \\
(23,9 \%)\end{array}$ & $\begin{array}{l}38 \\
(29,7 \%)\end{array}$ & $\begin{array}{l}43 \\
(28,6 \%)\end{array}$ & $\begin{array}{l}57 \\
(37,2 \%)\end{array}$ & $\begin{array}{l}63 \\
(36,6 \%)\end{array}$ & $\begin{array}{l}63 \\
(34,6 \%)\end{array}$ \\
\hline paysans & $\begin{array}{l}17 \\
(17,7 \%)\end{array}$ & $\begin{array}{l}25 \\
(22,7 \%)\end{array}$ & $\begin{array}{l}25 \\
(22,5 \%)\end{array}$ & $\begin{array}{l}24 \\
(21,2 \%)\end{array}$ & $\begin{array}{l}22 \\
(17,2 \%)\end{array}$ & $\begin{array}{l}45 \\
(30 \%)\end{array}$ & $\begin{array}{l}69 \\
(45,1 \%)\end{array}$ & $\begin{array}{l}78 \\
(45,3 \%)\end{array}$ & $\begin{array}{l}82 \\
(45 \%)\end{array}$ \\
\hline employés & $\begin{array}{l}24 \\
(25 \%)\end{array}$ & $\begin{array}{l}28 \\
(25,4 \%)\end{array}$ & $\begin{array}{l}28 \\
(25,2 \%)\end{array}$ & $\begin{array}{l}24 \\
(21,2 \%)\end{array}$ & $\begin{array}{l}27 \\
(21,1 \%)\end{array}$ & $\begin{array}{l}27 \\
(18 \%)\end{array}$ & $\begin{array}{l}8 \\
(5,2 \%)\end{array}$ & $\begin{array}{l}12 \\
(7 \%)\end{array}$ & $\begin{array}{l}20 \\
(11 \%)\end{array}$ \\
\hline $\begin{array}{l}\text { propriétaires } \\
\text { fonciers, } \\
\text { commerçants, } \\
\text { chefs d'entre- } \\
\text { prise, artisans }\end{array}$ & $\begin{array}{l}27 \\
(28,1 \%)\end{array}$ & $\begin{array}{l}28 \\
(25,4 \%)\end{array}$ & $\begin{array}{l}28 \\
(25,2 \%)\end{array}$ & $\begin{array}{l}26 \\
(23 \%)\end{array}$ & $\begin{array}{l}26 \\
(20,3 \%)\end{array}$ & $\begin{array}{l}18 \\
(12 \%)\end{array}$ & $\begin{array}{l}7 \\
(4,6 \%)\end{array}$ & $\begin{array}{l}9 \\
(5,2 \%)\end{array}$ & $\begin{array}{l}9 \\
(4,9 \%)\end{array}$ \\
\hline $\begin{array}{l}\text { serviteurs du } \\
\text { culte }\end{array}$ & $\begin{array}{l}1 \\
(1 \%)\end{array}$ & $\begin{array}{l}1 \\
(0,9 \%)\end{array}$ & - & - & $\begin{array}{l}2 \\
(1,5 \%)\end{array}$ & $\begin{array}{l}1 \\
(0,6 \%)\end{array}$ & & & \\
\hline $\begin{array}{l}\text { données } \\
\text { manquantes }\end{array}$ & $\begin{array}{l}4,2 \%) \\
(4,2 \%\end{array}$ & $\begin{array}{l}5 \\
(4,5 \%)\end{array}$ & $\begin{array}{l}3 \\
(2,7 \%)\end{array}$ & $\begin{array}{l}12 \\
(10,6 \%)\end{array}$ & $\begin{array}{l}13 \\
(10,1 \%)\end{array}$ & $\begin{array}{l}16 \\
(10,6 \%)\end{array}$ & $\begin{array}{l}12 \\
(7,8 \%)\end{array}$ & $\begin{array}{l}10 \\
(5,8 \%)\end{array}$ & $\begin{array}{l}8 \\
(4,4 \%)\end{array}$ \\
\hline
\end{tabular}


Tableau 3. Nombre de dirigeants du NKVD selon la nationalité

\begin{tabular}{|c|c|c|c|c|c|c|c|c|c|}
\hline Nationalité & 10.07.34 & 1.10 .36 & 1.03.37 & 1.07.37 & 1.01 .38 & 1.09 .38 & 1.07.39 & 1.01 .40 & 26.02 .41 \\
\hline Russes & $\begin{array}{l}30 \\
(31,2 \%)\end{array}$ & $\begin{array}{l}33 \\
(30 \%)\end{array}$ & $\begin{array}{l}35 \\
(31,5 \%)\end{array}$ & \begin{tabular}{|l|}
38 \\
$(33,6 \%)$
\end{tabular} & $\begin{array}{l}58 \\
(45,3 \%)\end{array}$ & $\begin{array}{l}85 \\
(56,6 \%)\end{array}$ & $\begin{array}{l}102 \\
(56,6 \%)\end{array}$ & $\begin{array}{l}111 \\
(64,5 \%)\end{array}$ & $\begin{array}{l}118 \\
(64,8 \%)\end{array}$ \\
\hline Juifs & $\begin{array}{l}37 \\
(38,5 \%)\end{array}$ & $\begin{array}{l}43 \\
(39,1 \%)\end{array}$ & $\begin{array}{l}42 \\
(37,8 \%)\end{array}$ & $\begin{array}{l}36 \\
(31,8 \%)\end{array}$ & $\begin{array}{l}35 \\
(27,3 \%)\end{array}$ & $\begin{array}{l}32 \\
(21,3 \%)\end{array}$ & $\begin{array}{l}6 \\
(3,9 \%)\end{array}$ & $\begin{array}{l}6 \\
(3,5 \%)\end{array}$ & $\begin{array}{l}10 \\
(5,5 \%)\end{array}$ \\
\hline Ukrainiens & $\begin{array}{l}5 \\
(5,2 \%)\end{array}$ & $\begin{array}{l}6 \\
(5,4 \%)\end{array}$ & $\begin{array}{l}6 \\
(5,4 \%)\end{array}$ & $\begin{array}{l}5 \\
(4,4 \%)\end{array}$ & $\begin{array}{l}4 \\
(3,1 \%)\end{array}$ & $\begin{array}{l}10 \\
(6,6 \%)\end{array}$ & $\begin{array}{l}19 \\
(12,4 \%)\end{array}$ & $\begin{array}{l}29 \\
(16,8 \%)\end{array}$ & $\begin{array}{l}28 \\
(15,4 \%)\end{array}$ \\
\hline Polonais & $\begin{array}{l}4 \\
(4,1 \%)\end{array}$ & $\begin{array}{l}5 \\
(4,5 \%)\end{array}$ & $\begin{array}{l}5 \\
(4,5 \%)\end{array}$ & $\begin{array}{l}4 \\
(3,5 \%)\end{array}$ & $\begin{array}{l}1 \\
(0,8 \%)\end{array}$ & $\begin{array}{l}1 \\
(0,7 \%)\end{array}$ & - & - & - \\
\hline Lettons & $\begin{array}{l}7 \\
(7,3 \%)\end{array}$ & $\begin{array}{l}9 \\
(8,2 \%)\end{array}$ & $\begin{array}{l}8 \\
(7,2 \%)\end{array}$ & $\begin{array}{l}7 \\
(6,2 \%)\end{array}$ & $\begin{array}{l}5 \\
(3,9 \%)\end{array}$ & - & - & - & $\begin{array}{l}1 \\
(0,5 \%)\end{array}$ \\
\hline Allemands & $\begin{array}{l}2 \\
(2,1 \%)\end{array}$ & $\begin{array}{l}2 \\
(1,8 \%)\end{array}$ & $\begin{array}{l}2 \\
(1,8 \%)\end{array}$ & $\begin{array}{l}2 \\
(1,7 \%)\end{array}$ & $\begin{array}{l}2 \\
(1,5 \%)\end{array}$ & $\begin{array}{l}1 \\
(0,6 \%)\end{array}$ & - & - & - \\
\hline Géorgiens & $\begin{array}{l}3 \\
(3,1 \%)\end{array}$ & $\begin{array}{l}4 \\
(3,6 \%)\end{array}$ & $\begin{array}{l}5 \\
(4,5 \%)\end{array}$ & $\begin{array}{l}4 \\
(3,5 \%)\end{array}$ & $\begin{array}{l}4 \\
(3,1 \%)\end{array}$ & $\begin{array}{l}5 \\
(3,3 \%)\end{array}$ & $\begin{array}{l}12 \\
(7,8 \%)\end{array}$ & $\begin{array}{l}12 \\
(7 \%)\end{array}$ & $\begin{array}{l}12 \\
(6,6 \%)\end{array}$ \\
\hline Arméniens & $\begin{array}{l}1 \\
(1 \%)\end{array}$ & $\begin{array}{l}1 \\
(0,9 \%)\end{array}$ & $\begin{array}{l}1 \\
(0,9 \%)\end{array}$ & $\begin{array}{l}1 \\
(0,9 \%)\end{array}$ & $\begin{array}{l}1 \\
(0,8 \%)\end{array}$ & $\begin{array}{l}1 \\
(0,6 \%)\end{array}$ & $\begin{array}{l}2 \\
(1,3 \%)\end{array}$ & $\begin{array}{l}2 \\
(1,2 \%)\end{array}$ & $\begin{array}{l}2 \\
(1,1 \%)\end{array}$ \\
\hline Azerbaïdjanais & $\begin{array}{l}1 \\
(1 \%)\end{array}$ & $\begin{array}{l}1 \\
(0,9 \%)\end{array}$ & $\begin{array}{l}1 \\
(0,9 \%)\end{array}$ & $\begin{array}{l}1 \\
(0,9 \%)\end{array}$ & - & - & - & - & - \\
\hline Biélorusses & $\begin{array}{ll}3 \\
(3,1 \%)\end{array}$ & $\begin{array}{l}2 \\
(1,8 \%)\end{array}$ & $\begin{array}{l}3 \\
(2,7 \%)\end{array}$ & $\begin{array}{l}3 \\
(2,6 \%)\end{array}$ & $\begin{array}{l}2 \\
(1,5 \%)\end{array}$ & $\begin{array}{l}3 \\
(2 \%)\end{array}$ & $\begin{array}{l}1 \\
(0,6 \%)\end{array}$ & $\begin{array}{l}3 \\
(1,7 \%)\end{array}$ & $\begin{array}{l}4 \\
(2,2 \%)\end{array}$ \\
\hline Autres & $\begin{array}{l}1 \\
(1 \%)\end{array}$ & $\begin{array}{l}1 \\
(0,9 \%)\end{array}$ & - & $\begin{array}{l}1 \\
(0,9 \%)\end{array}$ & $\begin{array}{l}1 \\
(0,8 \%)\end{array}$ & $\begin{array}{l}3 \\
(2 \%)\end{array}$ & $\begin{array}{l}1 \\
(0,6 \%)\end{array}$ & $\begin{array}{l}1 \\
(0,6 \%)\end{array}$ & $\begin{array}{l}3 \\
(1,6 \%)\end{array}$ \\
\hline $\begin{array}{l}\text { Données } \\
\text { manquantes }\end{array}$ & $\begin{array}{l}2 \\
(2,1 \%)\end{array}$ & $\begin{array}{l}2 \\
(1,8 \%)\end{array}$ & $\begin{array}{l}3 \\
(2,7 \%)\end{array}$ & $\begin{array}{l}11 \\
(9,7 \%)\end{array}$ & $\begin{array}{l}15 \\
(11,7 \%)\end{array}$ & $\begin{array}{l}9 \\
(6 \%)\end{array}$ & $\begin{array}{l}10 \\
(6,5 \%)\end{array}$ & $\begin{array}{l}8 \\
(4,6 \%)\end{array}$ & $\begin{array}{l}4 \\
(2,2 \%)\end{array}$ \\
\hline
\end{tabular}

Ce Tableau 3 montre clairement la tendance à l'accroissement de la représentation de la nation en titre (les Russes) à la direction du NKVD en 1937-1939. On y voit aussi l'orientation des purges du NKVD: les représentants des «nationalités étrangères » (inostrannye nacional'nosti) - selon la terminologie de 1937-1938 c'est-à-dire les Polonais, les Lettons et les Allemands ont été éliminés. Dans le contexte des événements survenus au cours des années suivantes, la brusque diminution du nombre des Juifs à la direction du NKVD est également significative ${ }^{16}$. La présence, jusque vers 1937-1938, dans les plus hautes instances du NKVD de telles proportions de Lettons, de Polonais et surtout de Juifs trouve en partie son explication dans les restrictions qui les affectaient directement sous l'ancien

16. Sur la totalité des employés des services de sécurité, la représentation des Juifs n'est pas très significative. Au 1er mars 1937, les Juifs représentaient $7 \%$ de l'effectif total du système du NKVD alors qu'au 1er janvier 1941 ils n'étaient plus que $4 \%$ (cf. GARF, f. 9401, op. 8, d. 43, 11 33-34 ; d. 64. 1. 24.). Dans la période d'avant-guerre, le principe de sélection des cadres du système de sécurité d'État à partir de la nomenclature a inévitablement abouti, au sein du NKVD, à retrouver le niveau général de représentation de tous les groupes nationaux du pays. Il n'existait pas encore de politique visant explicitement à évincer les Juifs des organes de la sécurité d'État. Il en alla tout autrement durant la période 1950-1953, lorsque les répressions au sein du MGB s'exercèrent exclusivement contre les Juifs. Dès la fin de l'année 1950, il ne restait que 1,5\% de Juifs dans le total des cadres opérationnels (Central'nyj Arhiv Federal'noj Služby Bezopasnosti-CA FSB, f. 4-os, op. 8, d. 11,11.310-341). 
régime. Le régime bolchevik, avec sa conception romantique de l'abolition des frontières nationales, a ouvert de nouvelles voies aux représentants de ces nations qui s'identifiaient avec le nouveau régime. Un nombre important d'entre eux s'inscrivit activement dans la vie politique et sociale soviétique et fit carrière après octobre 1917. L'effectif dirigeant du NKVD apparaît, en définitive, comme l'expression concentrée de cette tendance.

Cependant, sur la masse des employés des services de sécurité, ce phénomène était moins visible. Ainsi, selon les rapports de la section des cadres du NKVD, au

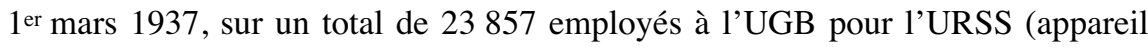
central non compris), on comptait 15570 Russes (65\%), 2509 Ukrainiens (11\%), 1776 Juifs (7\%), 980 Biélorusses (4\%), 426 Arméniens (1,8\%), 363 Tatares $(1,5 \%), 298$ Géorgiens $(1,2 \%), 256$ Lettons ( $1 \%), 144$ Polonais $(0,6 \%)$, les autres nationalités représentées étant proportionnellement insignifiantes ${ }^{17}$.

À la suite des purges, sur un total de 29737 employés dans les services de sécurité de l'URSS (non compris les employés de l'appareil central, des sections spéciales et des organes de transport), on comptait, au $1^{\text {er }}$ janvier 1941, 19528 Russes (66\%), 4877 Ukrainiens (16\%), 1273 Juifs (4\%), 797 Biélorusses $(2,7 \%), 525$ Arméniens (1,8\%), 391 Géorgiens (1,3\%). Le nombre de Polonais (6) et d'Allemands (10) était tout à fait insignifiant, tandis que les Lettons n'étaient même pas mentionnés ${ }^{18}$.

Tableau 4. Nombre de dirigeants du NKVD selon le niveau de formation

\begin{tabular}{|l|l|l|l|l|l|l|l|l|l|}
\hline $\begin{array}{c}\text { Niveau } \\
\text { de formation }\end{array}$ & $\mathbf{1 0 . 0 7 . 3 4}$ & $\mathbf{1 . 1 0 . 3 6}$ & $\mathbf{1 . 0 3 . 3 7}$ & $\mathbf{1 . 0 7 . 3 7}$ & $\mathbf{1 . 0 1 . 3 8}$ & $\mathbf{1 . 0 9 . 3 8}$ & $\mathbf{1 . 0 7 . 3 9}$ & $\mathbf{1 . 0 1 . 4 0}$ & $\mathbf{2 6 . 0 2 . 4 1}$ \\
\hline primaire & $\begin{array}{l}39 \\
(40,6 \%)\end{array}$ & $\begin{array}{l}38 \\
(34,5 \%)\end{array}$ & $\begin{array}{l}42 \\
(37,8 \%)\end{array}$ & $\begin{array}{l}41 \\
(36,3 \%)\end{array}$ & $\begin{array}{l}48 \\
(37,5 \%)\end{array}$ & $\begin{array}{l}64 \\
(42,6 \%)\end{array}$ & $\begin{array}{l}29 \\
(18,9 \%)\end{array}$ & $\begin{array}{l}31 \\
(18 \%)\end{array}$ & $\begin{array}{l}35 \\
(19,2 \%)\end{array}$ \\
\hline $\begin{array}{l}\text { secondaire et } \\
\text { second. } \\
\text { inachevé }\end{array}$ & $\begin{array}{l}40 \\
(41,6 \%)\end{array}$ & $\begin{array}{l}53 \\
(48,2 \%)\end{array}$ & $\begin{array}{l}49 \\
(44,1 \%)\end{array}$ & $\begin{array}{l}48 \\
(42,5 \%)\end{array}$ & $\begin{array}{l}56 \\
(43,7 \%)\end{array}$ & $\begin{array}{l}59 \\
(39,3 \%)\end{array}$ & $\begin{array}{l}55 \\
(36 \%)\end{array}$ & $\begin{array}{l}68 \\
(39,5 \%)\end{array}$ & $\begin{array}{l}81 \\
(44,5 \%)\end{array}$ \\
\hline $\begin{array}{l}\text { supérieur et } \\
\text { sup. inachevé }\end{array}$ & $\begin{array}{l}15 \\
(15,6 \%)\end{array}$ & $\begin{array}{l}16 \\
(14,5 \%)\end{array}$ & $\begin{array}{l}18 \\
(16,2 \%)\end{array}$ & $\begin{array}{l}13 \\
(11,5 \%)\end{array}$ & $\begin{array}{l}13 \\
(10,1 \%)\end{array}$ & $\begin{array}{l}15 \\
(10 \%)\end{array}$ & $\begin{array}{l}58 \\
(37,9 \%)\end{array}$ & $\begin{array}{l}65 \\
(37,8 \%)\end{array}$ & $\begin{array}{l}62 \\
(34 \%)\end{array}$ \\
\hline $\begin{array}{l}\text { données } \\
\text { manquantes }\end{array}$ & $\begin{array}{l}2 \\
(2,1 \%)\end{array}$ & $\begin{array}{l}3 \\
(2,7 \%)\end{array}$ & $\begin{array}{l}2 \\
(1,8 \%)\end{array}$ & $\begin{array}{l}11 \\
(9,7 \%)\end{array}$ & $\begin{array}{l}11 \\
(8,6 \%)\end{array}$ & $\begin{array}{l}12 \\
(8 \%)\end{array}$ & $\begin{array}{l}11 \\
(7,2 \%)\end{array}$ & $\begin{array}{l}8 \\
(4,6 \%)\end{array}$ & $\begin{array}{l}4 \\
(2,2 \%)\end{array}$ \\
\hline
\end{tabular}

Comme l'indique le Tableau 4, le niveau de formation des cadres de la Tchéka s'est élevé en 1939 et durant les années suivantes, alors que, jusqu'en 1938, le pourcentage des dirigeants du NKVD munis d'une formation élémentaire était très important. On voit clairement à qui Stalin confia la réalisation de la terreur de masse: il misa sur des exécutants à demi lettrés. Une fois passée la Grande Terreur, ces mêmes agents tombèrent sous le coup des arrestations. Il est intéressant de noter qu'au cours de cette période, le nombre de ceux dont l'enfance s'était déroulée dans des circonstances défavorables a augmenté au sein des instances dirigeantes du

17. GARF, f. 9401 , op. 8 , d. 43, 11. 33-34.

18. GARF, f. 9401 , op. 8, d. $64,1.24$. 
NKVD. Il s'agissait de personnes qui, dès leur jeune âge, avaient perdu l'un de leurs parents ou les deux, d'enfants abandonnés ou de vagabonds, exclus des établissements d'enseignement en raison de conflits avec les enseignants ou d'insoumission préméditée ou bien encore de mineurs ayant quitté le domicile parental à cause de conflits familiaux ou pour d'autres raisons. En 1934-1936, leur proportion était de 5 à $6 \%$ parmi les tchékistes dirigeants, en 1937, elle était de $8 \%$, et en 1938 de 12,7\% ; par la suite, en 1939-1940, elle est retombée brutalement à $6 \%$.

À l'évidence, les difficultés éprouvées dans l'enfance ne pouvaient que rejaillir sur le devenir de la personnalité du tchékiste. Qui plus est, pour la génération de tchékistes nés entre 1901 et 1905, il est probable que de telles circonstances aient favorisé leur admission au service de la VČK-OGPU - organe de justice sociale sommaire et « de vengeance prolétarienne ».

Les exemples biographiques abondent. Ainsi, le chef de l'UNKVD du territoire de l'Altaï, S. P. Popov, avait perdu sa mère prématurément et s'était enfui de chez lui à l'âge de 17 ans en raison de l'hostilité manifestée par sa belle-mère; le commissaire du peuple aux Affaires intérieures de Biélorussie, B. D. Berman, avait quitté la maison paternelle à l'âge de 11 ans ; le chef de l'UNKVD de l'oblast' de Riazan, S. Ja. Veršinin, avait été élevé dans une institution pour orphelins, celui de l'oblast' de Tula, S. I. Lebedev, était orphelin depuis l'âge de 8 ans; le vicecommissaire du peuple aux Affaires intérieures de l'URSS, L. M. Zakovskij, orphelin de père à l'âge de 4 ans, puis exclu de l'école, avait été laissé à l'influence de son frère aîné, un anarchiste. Les exemples pourraient ainsi être multipliés. C'est précisément sur ce type de parcours de tchékistes entrés en fonction dès leur jeune âge au service de Dzeržinskij que reposaient les enjeux des répressions staliniennes lors de la Grande Terreur.

L'un des objectifs (à en juger du moins d'après les résultats) des purges de la seconde moitié des années 1930 a été de renouveler les effectifs des fonctionnaires dirigeants de tous rangs et de relever le niveau de formation des responsables. De ce point de vue, le NKVD ne constituait nullement une exception. Il faut toutefois remarquer que, dans cet organe, la part de dirigeants ayant reçu une formation supé-

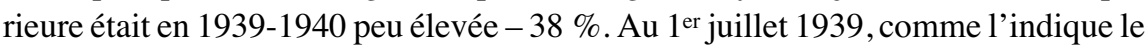
Tableau 4, un cinquième des dirigeants du NKVD n'avait reçu qu'une instruction élémentaire. Dans cette structure, l'expérience professionnelle, la fidélité et le dévouement étaient des qualités plus recherchées que le niveau de formation.

Les changements majeurs opérés dans l'encadrement des services de sécurité après la chute de Ežov se sont essentiellement manifestés par le relèvement du niveau de formation des dirigeants à tous les échelons du NKVD. Au $1^{\text {er }}$ juillet 1935, dans l'ensemble du système GUGB-UGB de l'Union, seules 1,6\% de personnes avaient reçu une formation supérieure, 23,8 \% - une formation secondaire et $74,6 \%$ - une formation primaire ${ }^{19}$. Au $1^{\text {er }}$ janvier $1941,26,1 \%$ du personnel de l'appareil central du NKVD étaient dotés d'une formation supérieure

19. GARF, f. 9401 , op. 8 , d. $40,1.16$. 
ou de niveau équivalent, 44,6\% d'une formation secondaire ou de niveau équivalent et 29,3\% d'une formation primaire ; dans les organes périphériques des services de sécurité, ces proportions étaient respectivement de 9,3\%,54,2\% et $36,5 \% 20$.

Les données présentées dans le Tableau 4 concernant le personnel dirigeant aux plus hauts échelons caractérisent beaucoup plus clairement et concrètement la situation. Il convient de noter, en particulier, la croissance du nombre de collaborateurs ayant une formation supérieure ou équivalente durant la période qui va du $1^{\text {er }}$ septembre 1938 au 1er juillet 1939, période au cours de laquelle leur proportion passe de 10 à $38 \%$ alors que, simultanément, la part des dirigeants dotés d'une formation primaire chute de $42,7 \%$ à $19 \%$. Autrement dit, les dirigeants à demilettrés du NKVD, mais bardés de grands mérites révolutionnaires et passés par le stage de tchékiste, ont accompli leur mission historique sous la direction de Ežov en conduisant « les opérations massives » d'arrestations et d'exécutions, à la suite de quoi ils furent considérés comme inutiles.

Il convient également de noter la tendance à la baisse du poids spécifique des personnes de formation supérieure en 1941 et simultanément la part croissante de celles ayant bénéficié d'une longue période de travail dans «les organes » (Cf. Tableaux 4 et 5). On observe une dépendance inversement proportionnelle entre le niveau de formation et l'expérience du travail dans les services de sécurité. Plus grande était l'expérience professionnelle et moins élevée était la formation, et réciproquement. Les nouveaux dirigeants, provenant du parti et des soviets, promus au NKVD vers la fin de 1938 et au début de 1939, n'ont pas tous réussi à s'accoutumer au système et à s'acquitter de leur tâche. Ils furent donc à nouveau remplacés par des tchékistes expérimentés, c'est-à-dire sans formation. Parmi ceux qui furent démis de leurs fonctions de dirigeants régionaux du NKVD en 1940 figurent notamment A. I. Starovojt (d'Odessa), I. F. Komarovskij (de Dnepropetrovsk), I. T. Jurčenko (de Nikolaevsk) et quelques autres. Leur courte formation effectuée dans les cours mensuels de l'École centrale du NKVD était clairement insuffisante pour mener à bien leur mission. Néanmoins, la majorité des promus en provenance du parti, arrivés pour un travail de direction à la Tchéka en 1938-1939 avec une préparation professionnelle minimale, ont réussi à faire carrière jusqu'au grade de général, ce qui constitue la meilleure illustration de la thèse bien connue de Lenin selon laquelle un bon communiste doit être un bon tchékiste.

Tableau 5. Nombre de dirigeants du NKVD en fonction de leur expérience tchékiste dans les « organes » de la VČK-OGPU-NKVD

\begin{tabular}{|c|c|c|c|c|c|c|c|c|c|}
\hline $\begin{array}{c}\text { Début du } \\
\text { travail à la } \\
\text { VČK-OGPU- } \\
\text { NKVD }\end{array}$ & $\mathbf{1 0 . 0 7 . 3 4}$ & $\mathbf{1 . 1 0 . 3 6}$ & $\mathbf{1 . 0 3 . 3 7}$ & $\mathbf{1 . 0 7 . 3 7}$ & $\mathbf{1 . 0 1 . 3 8}$ & $\mathbf{1 . 0 9 . 3 8}$ & $\mathbf{1 . 0 7 . 3 9}$ & $\mathbf{1 . 0 1 . 4 0}$ & $\mathbf{2 6 . 0 2 . 4 1}$ \\
\hline $1917-1920$ & $\begin{array}{l}68 \\
(70,8 \%)\end{array}$ & $\begin{array}{l}75 \\
(68,2 \%)\end{array}$ & $\begin{array}{l}73 \\
(65,8 \%)\end{array}$ & $\begin{array}{l}66 \\
(58,4 \%)\end{array}$ & $\begin{array}{l}62 \\
(48,4 \%)\end{array}$ & $\begin{array}{l}58 \\
(38,7 \%)\end{array}$ & $\begin{array}{l}8 \\
(5,2 \%)\end{array}$ & $\begin{array}{l}7 \\
(4 \%)\end{array}$ & $\begin{array}{l}8 \\
(4,4 \%)\end{array}$ \\
\hline
\end{tabular}

20. GARF, f. 9401 , op. 8, d. 64, 11. 6, 18. 


\begin{tabular}{|l|l|l|l|l|l|l|l|l|l|}
\hline $1921-1925$ & $\begin{array}{l}24 \\
(25 \%)\end{array}$ & $\begin{array}{l}26 \\
(23,6 \%)\end{array}$ & $\begin{array}{l}26 \\
(23,4 \%)\end{array}$ & $\begin{array}{l}25 \\
(22,1 \%)\end{array}$ & $\begin{array}{l}36 \\
(28,1 \%)\end{array}$ & $\begin{array}{l}48 \\
(32 \%)\end{array}$ & $\begin{array}{l}30 \\
(19,6 \%)\end{array}$ & $\begin{array}{l}37 \\
(21,5 \%)\end{array}$ & $\begin{array}{l}39 \\
(21,4 \%)\end{array}$ \\
\hline $1926-1930$ & $\begin{array}{l}3 \\
(3,1 \%)\end{array}$ & $\begin{array}{l}3 \\
(2,7 \%)\end{array}$ & $\begin{array}{l}4 \\
(3,6 \%)\end{array}$ & $\begin{array}{l}5 \\
(4,4 \%)\end{array}$ & $\begin{array}{l}12 \\
(9,4 \%)\end{array}$ & $\begin{array}{l}20 \\
(13,3 \%)\end{array}$ & $\begin{array}{l}19 \\
(12,4 \%)\end{array}$ & $\begin{array}{l}25 \\
(14,5 \%)\end{array}$ & $\begin{array}{l}33 \\
(18,1 \%)\end{array}$ \\
\hline $1931-1935$ & - & - & - & - & - & $\begin{array}{l}2 \\
(1,3 \%)\end{array}$ & $\begin{array}{l}15 \\
(9,8 \%)\end{array}$ & $\begin{array}{l}19 \\
(11 \%)\end{array}$ & $\begin{array}{l}23 \\
(12,6 \%)\end{array}$ \\
\hline $1936-1937$ & - & $\begin{array}{l}3 \\
(2,7 \%)\end{array}$ & $\begin{array}{l}6 \\
(5,4 \%)\end{array}$ & $\begin{array}{l}6 \\
(5,3 \%)\end{array}$ & $\begin{array}{l}7 \\
(5,4 \%)\end{array}$ & $\begin{array}{l}11 \\
7,3 \%)\end{array}$ & $\begin{array}{l}5 \\
(3,3 \%)\end{array}$ & $\begin{array}{l}7 \\
(4,1 \%)\end{array}$ & $\begin{array}{l}7 \\
(3,8 \%)\end{array}$ \\
\hline $1938-1939$ & - & - & - & - & - & - & $\begin{array}{l}67 \\
(43,8 \%)\end{array}$ & $\begin{array}{l}71 \\
(41,3 \%)\end{array}$ & $\begin{array}{l}70 \\
(38,4 \%)\end{array}$ \\
\hline $\begin{array}{l}\text { données } \\
\text { manquantes }\end{array}$ & $\begin{array}{l}1 \\
(1 \%)\end{array}$ & $\begin{array}{l}3 \\
(2,7 \%)\end{array}$ & $\begin{array}{l}2 \\
(1,8 \%)\end{array}$ & $\begin{array}{l}11 \\
(9,7 \%)\end{array}$ & $\begin{array}{l}11 \\
(8,6 \%)\end{array}$ & $\begin{array}{l}11 \\
(7,3 \%)\end{array}$ & $\begin{array}{l}9 \\
(5,9 \%)\end{array}$ & $\begin{array}{l}6 \\
(3,5 \%)\end{array}$ & $\begin{array}{l}2 \\
(1,1 \%)\end{array}$ \\
\hline
\end{tabular}

D'après le Tableau 5, on constate qu'en 1937-1938, il y avait dans le groupe dirigeant du NKVD un pourcentage élevé de personnes ayant pris leurs fonctions avant le milieu des années 1920. Jusqu'à présent, il était d'usage d'opposer « les vrais tchékistes » (les hommes de Dzeržinskij) à ceux appelés par Ežov ou Berija on ne sait d'où. Cependant, on voit bien que l'ossature de base de la direction du NKVD au $1^{\text {er }}$ janvier et au $1^{\text {er }}$ septembre 1938 était constituée par des personnes ayant rejoint les « organes » durant la période 1917-1925 (77\% et $71 \%$ respectivement), c'est-à-dire ayant servi sous Dzeržinskij. Ainsi est mise en évidence l'erreur courante concernant les hommes à l'aide desquels Staline a conduit les répressions de masse des années 1937-1938.

Tableau 6. Nombre de dirigeants du NKVD selon leur ancienneté au parti (RSDRP(b)-VKP(b))

\begin{tabular}{|c|c|c|c|c|c|c|c|c|c|}
\hline $\begin{array}{c}\text { Date d'entrée } \\
\text { au parti }\end{array}$ & 10.07.34 & 1.10 .36 & 1.03 .37 & 1.07.37 & 1.01 .38 & 1.09 .38 & 1.07.39 & 1.01 .40 & 26.02.41 \\
\hline avant 1917 & $\begin{array}{l}20 \\
(20,8 \%)\end{array}$ & $\begin{array}{l}16 \\
(14,5 \%)\end{array}$ & $\begin{array}{l}13 \\
(11,7 \%)\end{array}$ & $\begin{array}{l}11 \\
(9,7 \%)\end{array}$ & $\mid \begin{array}{l}5 \\
(3,9 \%)\end{array}$ & $\begin{array}{l}4 \\
(2,7 \%)\end{array}$ & - & - & - \\
\hline 1917 & $\begin{array}{l}19 \\
(19,8 \%)\end{array}$ & $\begin{array}{l}30 \\
(27,2 \%)\end{array}$ & $\begin{array}{l}31 \\
(27,9 \%)\end{array}$ & $\begin{array}{l}27 \\
(23,9 \%)\end{array}$ & $\begin{array}{l}21 \\
(16,4 \%)\end{array}$ & $\begin{array}{l}18 \\
(12 \%)\end{array}$ & $\begin{array}{l}3 \\
(1,9 \%)\end{array}$ & $\begin{array}{l}3 \\
(1,7 \%)\end{array}$ & $\begin{array}{l}4 \\
(2,2 \%)\end{array}$ \\
\hline $1918-1920$ & $\begin{array}{l}48 \\
(50 \%)\end{array}$ & $\begin{array}{l}50 \\
(45,4 \%)\end{array}$ & $\begin{array}{l}54 \\
(48,6 \%)\end{array}$ & $\begin{array}{l}52 \\
(46 \%)\end{array}$ & $\begin{array}{l}66 \\
(51,5 \%)\end{array}$ & $\begin{array}{l}73 \\
(48,7 \%)\end{array}$ & $\begin{array}{l}22 \\
(14,4 \%)\end{array}$ & $\begin{array}{l}23 \\
(13,4 \%)\end{array}$ & $\begin{array}{l}23 \\
(12,6 \%)\end{array}$ \\
\hline 1921-1924 & $\begin{array}{l}6 \\
(6,2 \%)\end{array}$ & $\begin{array}{l}6 \\
(5,4 \%)\end{array}$ & $\begin{array}{l}6 \\
(5,4 \%)\end{array}$ & $\begin{array}{l}4 \\
(3,5 \%)\end{array}$ & $\begin{array}{l}6 \\
(4,7 \%)\end{array}$ & $\begin{array}{l}11 \\
(7,3 \%)\end{array}$ & $\begin{array}{l}9 \\
(5,9 \%)\end{array}$ & $\begin{array}{l}12 \\
(7 \%)\end{array}$ & $\begin{array}{l}12 \\
(7 \%)\end{array}$ \\
\hline $1925-1928$ & $\begin{array}{l}1 \\
(1 \%)\end{array}$ & $\begin{array}{l}5 \\
(4,5 \%)\end{array}$ & $\begin{array}{l}4 \\
(3,6 \%)\end{array}$ & $\begin{array}{l}5 \\
(4,4 \%)\end{array}$ & $\begin{array}{l}15 \\
(11,7 \%)\end{array}$ & $\begin{array}{l}24 \\
(16 \%)\end{array}$ & $\begin{array}{l}66 \\
(43,1 \%)\end{array}$ & $\begin{array}{l}71 \\
(41,3 \%)\end{array}$ & $\begin{array}{l}80 \\
(44 \%)\end{array}$ \\
\hline 1929-1932 & - & - & - & $\begin{array}{l}2 \\
(1,8 \%)\end{array}$ & $\begin{array}{l}2 \\
(1,6 \%)\end{array}$ & $\begin{array}{l}6 \\
(4 \%)\end{array}$ & $\begin{array}{l}38 \\
(24,8 \%)\end{array}$ & $\begin{array}{l}45 \\
(26,1 \%)\end{array}$ & $\begin{array}{l}47 \\
(25,8 \%)\end{array}$ \\
\hline $1933-1936$ & - & - & - & - & - & - & $\begin{array}{l}1 \\
(0,6 \%)\end{array}$ & $\begin{array}{l}1 \\
(0,6 \%)\end{array}$ & $\begin{array}{l}2 \\
(1,1 \%)\end{array}$ \\
\hline 1937 et après & - & - & - & - & $\begin{array}{l}1 \\
(0,8 \%)\end{array}$ & $\begin{array}{l}2 \\
(1,3 \%)\end{array}$ & $\begin{array}{l}3 \\
(2 \%)\end{array}$ & $\begin{array}{l}9 \\
(5,2 \%)\end{array}$ & $\begin{array}{l}9 \\
(4,9 \%)\end{array}$ \\
\hline $\begin{array}{l}\text { données } \\
\text { manquantes }\end{array}$ & $\begin{array}{l}2 \\
(2,1 \%)\end{array}$ & \begin{tabular}{|l}
3 \\
$(2,7 \%)$
\end{tabular} & $\begin{array}{l}3 \\
(2,7 \%)\end{array}$ & $\begin{array}{l}12 \\
(10,6 \%)\end{array}$ & $\begin{array}{l}12 \\
(9,4 \%)\end{array}$ & $\begin{array}{l}12 \\
(8 \%)\end{array}$ & $\begin{array}{l}11 \\
(7,2 \%)\end{array}$ & $\begin{array}{l}8 \\
(4,6 \%)\end{array}$ & $\begin{array}{l}5 \\
(2,7 \%)\end{array}$ \\
\hline
\end{tabular}


Tableau 7. Nombre de dirigeants du NKVD selon leur appartenance passée à différents partis ou mouvements

\begin{tabular}{|c|c|c|c|c|c|c|c|c|c|}
\hline $\begin{array}{c}\text { Parti, } \\
\text { mouvement }\end{array}$ & 10.07 .34 & 1.10 .36 & 1.03.37 & 1.07.37 & 1.01 .38 & 1.09 .38 & 1.07.39 & 1.01 .40 & 26.02 .41 \\
\hline parti SR & $\begin{array}{l}7 \\
(7,3 \%)\end{array}$ & $\begin{array}{l}5 \\
(4,5 \%)\end{array}$ & $\begin{array}{l}6 \\
(5,4 \%)\end{array}$ & $\begin{array}{l}6 \\
(5,3 \%)\end{array}$ & $\begin{array}{l}6 \\
(4,7 \%)\end{array}$ & $\begin{array}{l}5 \\
(3,3 \%)\end{array}$ & - & - & - \\
\hline $\begin{array}{l}\operatorname{RSDRP}(m) \text { et } \\
\operatorname{RSDRP}(\mathrm{i})^{*}\end{array}$ & $\begin{array}{l}5 \\
(5,2 \%)\end{array}$ & $\begin{array}{l}6 \\
(5,4 \%)\end{array}$ & $\begin{array}{l}6 \\
(5,4 \%)\end{array}$ & $\begin{array}{l}3 \\
(2,6 \%)\end{array}$ & - & - & - & - & - \\
\hline anarchiste & $\begin{array}{l}7 \\
(7,3 \%)\end{array}$ & $\begin{array}{l}3 \\
(2,7 \%)\end{array}$ & $\begin{array}{l}3 \\
(2,7 \%)\end{array}$ & $\begin{array}{l}3 \\
(2,6 \%)\end{array}$ & $\begin{array}{l}2 \\
(1,6 \%)\end{array}$ & - & - & - & - \\
\hline Bund & $\begin{array}{l}3 \\
(3,1 \%)\end{array}$ & $\begin{array}{l}3 \\
(2,7 \%)\end{array}$ & $\begin{array}{l}3 \\
(2,7 \%)\end{array}$ & $\begin{array}{l}2 \\
(1,8 \%)\end{array}$ & $\begin{array}{l}2 \\
(1,6 \%)\end{array}$ & - & - & - & - \\
\hline Poale Zion & $\begin{array}{l}5 \\
(5,2 \%)\end{array}$ & $\begin{array}{l}5 \\
(4,5 \%)\end{array}$ & $\begin{array}{l}5 \\
(4,5 \%)\end{array}$ & $\begin{array}{l}4 \\
(3,5 \%)\end{array}$ & $\begin{array}{l}3 \\
(2,3 \%)\end{array}$ & $\begin{array}{l}1 \\
(0,6 \%)\end{array}$ & - & - & - \\
\hline Dachnak & - & - & - & - & $\begin{array}{l}1 \\
(0,7 \%)\end{array}$ & $\begin{array}{l}1 \\
(0,6 \%)\end{array}$ & - & - & - \\
\hline Musavat & - & - & - & - & - & $\begin{array}{l}1 \\
(0,7 \%)\end{array}$ & $\begin{array}{l}1 \\
(0,6 \%)\end{array}$ & $\begin{array}{l}1 \\
(0,6 \%)\end{array}$ & $\begin{array}{l}1 \\
(0,5 \%)\end{array}$ \\
\hline $\begin{array}{l}\text { mouvement } \\
\text { Blanc }\end{array}$ & $\begin{array}{l}3 \\
(3,1 \%)\end{array}$ & $\begin{array}{l}2 \\
(1,8 \%)\end{array}$ & $\begin{array}{l}3 \\
(2,7 \%)\end{array}$ & $\begin{array}{l}4 \\
(3,5 \%)\end{array}$ & $\begin{array}{l}3 \\
(2,3 \%)\end{array}$ & $\begin{array}{l}2 \\
(1,3 \%)\end{array}$ & - & - & - \\
\hline $\begin{array}{l}\text { sans passé } \\
\text { communiste }\end{array}$ & $\begin{array}{l}30 \\
(31,2 \%)\end{array}$ & $\begin{array}{l}24 \\
(21,8 \%)\end{array}$ & $\begin{array}{l}25 \\
(22,5 \%)\end{array}$ & $\begin{array}{l}22 \\
(19,5 \%)\end{array}$ & $\begin{array}{l}17 \\
(13,3 \%)\end{array}$ & $\begin{array}{l}10 \\
(6,7 \%)\end{array}$ & $\begin{array}{l}1 \\
(0,6 \%)\end{array}$ & $\begin{array}{l}1 \\
(0,6 \%)\end{array}$ & $\begin{array}{l}1 \\
(0,5 \%)\end{array}$ \\
\hline
\end{tabular}

* Mencheviks et « Internationalistes ».

Les données présentées dans les Tableaux 2, 3 et 7 , fournissent de manière évidente les motifs des répressions menées à l'encontre des dirigeants du NKVD. Dans le contexte du début des répressions de masse, les origines sociales étrangères, l'appartenance à des nations limitrophes, à l'époque considérées comme ennemies, et enfin l'appartenance passée à des partis ou à des courants politiques non bolcheviks ont immanquablement entrâ̂né des arrestations ou en ont accru la probabilité. Se pose cependant une question de logique dans le fait d'exercer des purges au sein même de l'appareil punitif appelé à conduire la répression la plus large possible. La purge interne au NKVD n'a-t-elle pas entravé la réussite des répressions à l'échelle de la société dans son ensemble ? À la différence des campagnes d'arrestations et de déportations précédentes qui touchaient des catégories de population strictement définies, la tendance générale des répressions de 1937-1938 a été d'englober toutes les couches de la société soviétique sans exception, et de toucher l'ensemble des structures de l'appareil du parti et de l'État. Dans ces conditions de «purge généralisée » l'appareil du NKVD ne pouvait constituer une exception.

La vaste campagne de révélation de la «conspiration du bloc de droite trotskiste » contre le gouvernement de Stalin, lancée par N. I. Ežov lors du plénum du Comité central du VKP(b) de juin 1937, exigeait des victimes jusqu'au sein du NKVD. C'était l'achèvement logique, inéluctable, de «l'affaire Kirov », dont le meurtre s'expliquait désormais non pas par la négligence de tchékistes isolés mais par l'existence d'un large «complot» au cœur du NKVD, conduit par Jagoda. Dans ce contexte, Stalin estimait que l'appareil même du NKVD dans son 
ensemble s'était ossifié. La rigidité des méthodes, la crainte des changements et la peur de perdre son fauteuil de direction avaient provoqué une forme d'inertie dans le travail et de repli de l'institution sur elle-même. Stalin avait bien senti qu'une sérieuse secousse du corps dirigeant était devenue indispensable et qu'il fallait opérer des mutations dans l'encadrement. Plus que d'autres facteurs, «l'obstruction » (zasorennost') des cadres de la Tchéka l'inquiétait particulièrement. Parmi ces derniers, la proportion d'agents au passé «non communiste», originaires de classes « étrangères » ou « ennemies » (cf. Tableau 2) était jugée trop importante, de même que celle des cadres appartenant à des nationalités «non titulaires » de l'URSS (cf. Tableau 3), et inscrits dans le passé à différents partis ou mouvements non bolcheviks ( cf. Tableau 7), ce qui, au milieu des années 1930, était considéré comme un facteur aggravant.

Le pourcentage de dirigeants tchékistes qui avaient appartenu dans leur jeunesse à des partis ou à des mouvements anti-léninistes, même s'ils étaient socialistes, ou qui, plus encore, avaient servi la cause des Blancs, était extraordinairement élevé en 1934. De toute évidence, ces tchékistes constituaient les premiers candidats aux arrestations dans la vague de purges entreprises au sein même du NKVD. Cela étant, les répressions avaient leur propre logique, et, du temps où Ežov était aux commandes, certains en réchappèrent en parvenant à taire les aspects douteux de leur passé, d'autres en étant particulièrement proches et appréciés de Ežov. Leur carrière s'acheva lorsque Berija prit la tête du NKVD. Comme l'indique le Tableau 7 , il ne restait sous sa direction qu'un seul homme ayant pris part au mouvement Musavat-Berjia en personne

Tous ces facteurs conjugués ont prédéterminé la purge de l'ensemble de l'appareil du NKVD. Les arrestations pratiquées dans les rangs des tchékistes ont, en soi, créé l'atmosphère indispensable au renforcement des « organes » dans la préparation des répressions et leur gestion. En réalité, le personnel de niveau intermédiaire du NKVD - les chefs adjoints des directions et les chefs de départements des directions régionales, qui avaient des perspectives de promotion rapide, pouvaient, sans aucun égard, briser les autorités avec véhémence et enthousiasme, procéder sans encombres aux arrestations de hauts dirigeants du parti et, ainsi, «faire feu sur les états-majors ». Ils n'avaient pas de cohésion, ne faisaient pas corps avec les cadres du parti et des soviets comme c'était le cas des dirigeants locaux du NKVD à l'époque de Jagoda. Les purges au sommet touchèrent avec la même intensité le système interne du NKVD dans lequel Stalin cherchait à se débarrasser des « clans » forts et des groupes tchékistes en les cassant, en brisant les liens existant de longue date, en atomisant l'oligarchie dirigeante.

Tableau 8. Nombre de dirigeants du NKVD arrêtés

\begin{tabular}{|l|r|r|r|r|r|r|r|}
\hline & 1934 & 1935 & 1936 & 1937 & 1938 & 1939 & 1940 \\
\hline janvier & & & & & 4 & 22 & \\
\hline février & & & & 1 & 3 & 4 & \\
\hline mars & & & & 4 & 1 & 2 & \\
\hline
\end{tabular}




\begin{tabular}{|l|r|r|r|r|r|r|c|}
\hline & 1934 & 1935 & 1936 & 1937 & 1938 & 1939 & 1940 \\
\hline avril & & & & 5 & 9 & 7 & 1 \\
\hline mai & & & & 4 & 3 & 2 & \\
\hline juin & & & & 6 & 5 & 3 & 1 \\
\hline juillet & & & & 9 & 5 & & \\
\hline août & & & & 17 & & 1 & \\
\hline septembre & & & & 3 & 12 & & \\
\hline octobre & & & & 8 & 17 & & \\
\hline novembre & & & 1 & 5 & 20 & & \\
\hline décembre & 1 & & & 2 & 24 & & \\
\hline sans date précise & & & 1 & 7 & 4 & 16 & 1 \\
\hline total de l'année & 1 & aucun & 2 & 71 & 107 & 57 & 3 \\
\hline
\end{tabular}

$\mathrm{n}=241$.

Le Tableau 8 met en évidence l'échelle des purges effectuées parmi les cadres de la direction du NKVD. Sur 322 dirigeants des organes du NKVD dans les républiques, de ses directions dans les kraja et les oblasti et des subdivisions de l'appareil central, qui ont occupé ces postes à différents moments entre juillet 1934 et septembre 1938, 241 personnes ont été arrêtées (soit près de $75 \%$ ). Le pic des répressions se situe dans les premiers mois où Berija a pris les commandes du NKVD. Par comparaison, la purge conduite par Ežov semble on ne peut plus modeste. Notons également la réduction des purges du NKVD au début de l'année 1938, Ežov ayant dû estimer, après l'arrestation des principaux partisans de Jagoda, que les cadres restants étaient « sous contrôle ».

Si l'on considère le début de la grande campagne d'arrestations au NKVD (Tableau 8), durant la période 1934-1936, ce qui caractérise les arrestations c'est leur aspect fortuit ; elles ne correspondent pas encore à une purge politique réfléchie. Ainsi, en décembre 1934, fut arrêté le chef de la direction de Leningrad, F. D. Medved', accusé de négligence criminelle dans l'affaire liée au meurtre de Kirov. En 1935, on n'enregistre aucune arrestation de dirigeants tchékistes. En 1936, les victimes furent le chef de la direction de Karakalpakie, F. D. Ul'drik, pour abus de pouvoir et, en novembre 1936, le chef adjoint de la direction de Saratov, I. I. Sosnovskij, accusé d'espionnage au profit de la Pologne. L'arrestation de ce dernier fut justement considérée comme le présage des importantes purges à venir. En février 1937, eut lieu l'arrestation du commissaire du peuple aux Affaires intérieures de Biélorussie, G. A. Molčanov (autrefois à la tête du SPO GUGB - Special'nyj Političeskij Otdel, Glavnoe Upravlenie Gosudarstvennoj Bezopasnosti), et, à compter de mars 1937, à la suite d'une résolution spéciale prise en février-mars par le plénum du Comité central du parti, les purges du NKVD prirent un caractère régulier et ciblé. 
Tableau 9. Nombre de jugements de dirigeants du NKVD

\begin{tabular}{|l|c|c|c|c|c|}
\hline & 1937 & 1938 & 1939 & 1940 & 1941 \\
\hline janvier & & 3 & & 30 & \\
\hline février & & 12 & 30 & 17 & 1 \\
\hline mars & & 3 & 11 & 2 & 1 \\
\hline avril & & 2 & 1 & 1 & \\
\hline mai & & 2 & 7 & 4 & 3 \\
\hline juin & 4 & 3 & 3 & 2 & 1 \\
\hline juillet & & 4 & & 3 & 11 \\
\hline août & 4 & 11 & 1 & & \\
\hline septembre & 6 & 3 & 4 & 1 & \\
\hline octobre & 5 & & 2 & & \\
\hline novembre & 9 & & 3 & 1 & \\
\hline décembre & 3 & & 3 & 3 & \\
\hline sans date précise & 1 & 2 & 1 & 1 & \\
\hline total de l'année & 32 & 45 & 66 & 65 & 17 \\
\hline
\end{tabular}

$\mathrm{n}=225$.

Le Tableau 9 met en évidence les principales campagnes de jugements des tchékistes arrêtés. Le fait que le total des jugements soit moins élevé que celui des arrestations (cf. Tableau 8) s'explique sans doute en partie parce que nos données sont incomplètes mais surtout parce que tous les tchékistes arrêtés ne survivaient pas jusqu'au verdict, comme ce fut le cas, par exemple, du vice-commissaire du peuple aux Affaires intérieures, M. I. Ryžov, du commissaire du peuple aux Affaires intérieures d'Arménie, V. V. Hvorostjan, du chef de la direction de l'oblast' de Čita, G. S. Horhorin. Certains ne survécurent même pas jusqu'à leur procès, tels le commissaire du peuple aux Affaires intérieures de Kalmykie, P. G. Ozerkin, et l'ancien chef de la direction de l'oblast' de l'Ob'-Irtyš, S. V. Zdorovcev ; d'autres furent tout simplement libérés lors de la révision des procès en 1939. Par rapport à l'époque de Ežov, il est clair qu'un nombre beaucoup plus important de dirigeants tchékistes fut jugé du temps de Berija, même si certains d'entre eux avaient été arrêtés sous Ežov. Mais, durant les premiers mois de son activité à la Loubianka, Berija ne s'est pas montré très empressé de les faire passer en jugement, ayant l'espoir d'obtenir d'eux des dépositions contre Ežov et ses plus proches collaborateurs. Ainsi s'explique l'absence de condamnation entre octobre 1938 et janvier 1939. La série des procès n'a repris qu'en février-mars 1939. Il arrivait que le laps de temps entre l'arrestation et le jugement ne soit que de quelques mois. Néanmoins, le plus souvent, l'instruction était longue - un an et plus. En 1937-1938, les sentences contre les dirigeants tchékistes (à la peine capitale dans un nombre écrasant de cas) étaient, plus ou moins régulièrement, établies à l'avance par Stalin et 
ses proches. Cette prédétermination des condamnations est également caractéristique de la période 1939-1941 même si les procès de ces années-là relevaient davantage d'une campagne. Le nombre considérable de condamnés enregistrés au tout début de 1939 et de 1940 s'explique par l'existence d'ordres directs de Stalin et du Politbjuro imposant des audiences secrètes au Collège de guerre (Voennaja Kollegija) de la Cour suprême de l'URSS pour les affaires concernant les dirigeants du NKVD (il en fut de même en juillet 1941).

L'appréciation à porter sur les chiffres de la répression dans l'ensemble du réseau du NKVD mérite un commentaire particulier. Combien y a-t-il eu au total de victimes tchékistes des purges staliniennes? D'après les statistiques internes du NKVD pour la période du 1er octobre 1936 au 15 août 1938, qui correspond à l'ère Ežov, 2273 collaborateurs de l'UGB (Sécurité d'État) furent arrêtés dans tout le pays ; 1862 d'entre eux seulement furent arrêtés pour crimes contre-révolutionnaires, les autres l'ayant été pour crimes de droit commun (désorganisation du travail, discrédit jeté sur les services et corruption des mœurs) ${ }^{21}$. Dans ce nombre total d'arrestations ne sont pas inclus: les hommes des troupes de l'intérieur, les gardes-frontières, les pompiers, les miliciens, les employés du Goulag et d'autres services auxiliaires qui furent poursuivis à la même époque. Si l'on compare le chiffre concernant les collaborateurs des services de sécurité (1 862 arrestations) avec la liste approximative du nombre d'agents de ce service, soit 25000 personnes au $1^{\text {er }}$ mars 1937, on constate que seulement 7,5\% des employés de la sécurité furent arrêtés sous l'accusation de crimes contre-révolutionnaires, ce qui n'est pas si considérable, surtout si l'on a en tête qu'à la fin des années 1980, le chiffre de 20000 tchékistes victimes des répressions était officiellement avancé22. Ce dernier chiffre a eu cours jusque dans la littérature historique et même dans des documents scientifiques. Il requiert toutefois quelques éclaircissements. En effet, ce n'est pas un hasard si au cours de la «perestroïka » de Gorbačev, l'accent a été mis sur les tchékistes victimes des répressions, car il s'agissait de conforter la thèse selon laquelle les répressions staliniennes, soi disant étrangères à la nature du régime soviétique, avaient rencontré une opposition au sein même de l'appareil du NKVD menée par les tchékistes « de Dzeržinskij » que Stalin dut réprimer.

En outre, il n'est jamais précisé à quelle période se rapportent les répressions et qui en a particulièrement souffert. En réalité, ce chiffre $(20000)$, conformément aux données des archives, concerne la période 1933-1939 et comprend non seulement toutes les catégories de collaborateurs du système de l'OGPU-NKVD (depuis les gardes-frontières jusqu'aux surveillants de camps en passant par les appelés des troupes du NKVD), mais également toutes sortes de criminels y compris les dangereux criminels de droit commun. Le nombre de collaborateurs du système de l'OGPU-NKVD arrêtés au cours de ces années se répartit de la manière suivante: en 1933 - 738 personnes; en $1934-2860$; en $1935-6249$; en $1936-1945$; en

21. CA FSB, f. 3, op. 5, d. 996,1. 189.

22. V. M. Čebrikov, « Peretrojka i rabota čekistov » (La perestroïka et le travail des tchékistes), Pravda, 2 septembre 1988. 
$1937-3837$; en $1938-5625$; en 1939 - 1364 . Au total, de 1933 à 1939, 22618 personnes ${ }^{23}$.

Comme on le voit, en 1937-1938, au total 9462 collaborateurs du NKVD furent arrêtés. Toutefois, ce chiffre comprend une écrasante majorité de miliciens, de pompiers, d'hommes de troupes du NKVD, dont des hommes du rang (ils étaient assez nombreux et faisaient partie de l'encadrement du NKVD), d'employés du Goulag, de fonctionnaires de l'état civil, etc., mais pas de collaborateurs des services de sécurité - agents directs de la terreur. De plus, la majorité d'entre eux avaient été condamnés pour des crimes de droit commun et non pour des motifs politiques.

Comme on l'a déjà été précisé, 25000 personnes environ travaillaient dans les services de sécurité de la GUGB-UGB à l'époque de Ežov. Relativement à cette masse d'employés, le chiffre avancé de 20000 personnes qui auraient subi les répressions semble tout simplement insensé. Il est clair qu'elles n'auraient pas toutes pu faire l'objet de répression, à supposer même qu'elles se fussent autoréprimées.

La purge engagée au sein des cadres opérationnels de la Tchéka a pris un tour planifié en 1939. Mais cette purge engagée sous Berija, qui commença d'ailleurs dès l'automne 1938 et qui avait pour objet de « restaurer la légalité socialiste », ne peut vraisemblablement pas être considérée comme une répression politique à l'encontre de "tchékistes honnêtes ». Il s'agissait, pour l'essentiel, de la première révolution professionnelle dans les organes de la sécurité d'État, orientée vers le renouvellement radical des effectifs, comme le montrent les révocations massives dans les organes du NKVD qui ont constitué le fondement de la purge: en 1939, 7372 collaborateurs ont été destitués $(22,9 \%$ selon les effectifs déclarés de tchékistes opérationnels). Sur ce total, 973 collaborateurs de Ežov, meneurs les plus odieux de la terreur de masse, tombèrent sous le coup des arrestations ${ }^{24}$. Si l'on prend également en compte les arrestations de la fin de l'année 1938, il s'avère que, selon certaines données, la somme totale des collaborateurs des services de sécurité arrêtés du temps de Berija à partir de la fin 1938 et pendant toute l'année 1939 s'élève à 1364 personnes. Des dernières générations d'employés en poste sous Ežov, $34 \%$ de ceux en poste au $1^{\text {er }}$ janvier 1938 et $35 \%$ de ceux en poste au $1^{\text {er }}$ septembre 1938 ont été arrêtés dans la deuxième moitié de l'année 1938, ainsi que $20 \%$ et $29 \%$ respectivement durant la première moitié de l'année 1939. Soit une écrasante majorité. Ainsi, s'agissant de la couche dirigeante du NKVD, la purge de Berija a été beaucoup plus importante que celle de Ežov.

La «Grande Terreur» aboutit au quasi doublement des effectifs de collabora-

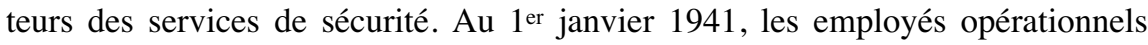
étaient au nombre de 3656 au sein de l'appareil central du NKVD de l'URSS, de 29737 dans les organes locaux, de 7901 dans le Département spécial (Osobyj otdel) du NKVD (renseignement militaire), de 3804 dans les services du transport

23. CA FSB, f. 3-os, op. 6, d. 33, 1. 4 .

24. GARF, f. 9401 , op. 8, d. 51,1.2. 
du NKVD et de 1118 personnes dans le réseau des écoles du NKVD (École Supérieure et inter-territoriale), soit un total de 46216 personnes $^{25}$.

Comme l'indiquent les rapports statistiques de la section des cadres du NKVD, au $1^{\text {er }}$ janvier 1941, la majorité des nouvelles recrues tchékistes (soit 53,7\%) pouvaient faire état d'une expérience de 3 ans au plus dans les services, étant entrés au NKVD en 1938-193926. Fin 1938, les principes de formation des cadres des services de sécurité furent modifiés en profondeur, en particulier pour les postes de dirigeants. La plupart de ceux qui ont été promus au niveau des directions du NKVD dans les républiques, les territoires et les régions, sont arrivés en poste sur décision du Comité central du VKP(b) après avoir rempli des fonctions de cadres moyens au sein du parti, des soviets, des établissements d'enseignement supérieur ou de l'industrie.

Les nouveaux principes de formation du personnel d'encadrement des services de sécurité et l'ampleur des changements sont spectaculaires. Au cours de la seule année 1939, 14506 personnes furent embauchées à des postes opérationnels dans l'appareil du NKVD (soit 45,1\% de l'ensemble des effectifs). Ils furent recrutés comme suit:

- 11062 agents $(76,3 \%)$ en provenance des organes des komsomols, du parti et des écoles du NKVD ;

- 1332 agents $(9,7 \%)$ ont été transférés d'emploi non opérationnels d'autres départements et d'autres directions à des postes opérationnels ;

-1129 agents $(7,7 \%)^{27}$ ont été promus de leurs postes technico-administratifs vers des emplois opérationnels.

S'agissant des différentes phases de purges de l'encadrement du NKVD et des données quantitatives y affairant, les conclusions à tirer peuvent, à première vue, sembler paradoxales et en contradiction avec les représentations dominantes. Ainsi, jusqu'alors, on admettait couramment que la purge majeure du NKVD avait débuté dès l'arrivée de Ežov à la direction des « organes ». Il est certain que le début des arrestations de tchékistes formés sous Jagoda se situe dès la première moitié de l'année 1937, mais ce fait n'a pas modifié en profondeur les caractéristiques sociopolitiques du personnel d'encadrement. Pour tous les indicateurs (en pourcentage) fournis au cours de la période 1937-1938, les variations observées semblent correspondre à une évolution naturelle. Par contre, l'arrivée de Berija à la tête du NKVD est marquée par les variations très brutales des différents indicateurs qui témoignent de la purge entreprise. C'est dire que cette période reste caractérisée par d'importants bouleversements dans l'effectif dirigeant. Pour s'en convaincre, il suffit de rappeler l'ampleur des changements de cadres sous Berija: du $1^{\text {er }}$ septembre 1938 au $1^{\text {er }}$ juillet 1939 presque tous les dirigeants du NKVD des républiques et des oblasti de l'URSS ont été remplacés. Ceux qui firent exception à la règle sont $\mathrm{T}$. M. Boř̌čev, commissaire du peuple aux Affaires intérieures de

25. GARF, f. 9401 , op. 8 , d. $64,11.1,17,25,40,49$.

26. Ibid.,1. 18.

27. GARF, f. 9401 ,op. 8 , d. $50,1.1$. 
Turkménie, nommé à ce poste en juillet 1938 et V. A. Tkačev, commissaire du peuple aux Affaires intérieures de la République autonome de Bouriatie-Mongolie, ce dernier n'ayant été maintenu en fonction que par inadvertance puisqu'il fut en rapidement démis, puis arrêté et fusillé en 1941. Seul, T. M. Borščev, qui avait travaillé dans les années 20 pour les services de l'OGPU de Transcaucasie, était un homme de Berija au plein sens de terme, et c'est précisément ce qui allait le perdre par la suite car il fut, lui aussi, fusillé en 1956.

La période suivante (1941-1951) fut plus ou moins stable du point de vue de la composition socio-politique des effectifs d'encadrement dans les services de sécurité. Il ne s'est pas produit de brusques changements, ni de purges significatives ou d'autres reflux importants de cadres. La tendance de cette période a été, au contraire, celle d'un accroissement constant d'effectifs dans les appareils (tant à l'échelon central que dans les organes périphériques).

En février 1941, le commissariat du peuple à la Sécurité d'État (NKGB) devint un organisme indépendant, détaché des effectifs du NKVD. Les organes militaires du contre-espionnage ne furent pas inclus dans ses effectifs, ils furent transmis au commissariat du peuple à la Défense. Ceci explique le nombre relativement faible de cadres opérationnels du NKGB au moment de sa création. Ainsi, au 21 mai 1941, la nomenclature des postes du personnel d'encadrement du NKGB d'URSS comprenait 6722 postes (depuis les chefs adjoints des sections indépendantes jusqu'aux responsables des directions et aux commissaires du peuple à la Sécurité d'État) pour tous les organes territoriaux (hors appareil central). L'existence du NKGB en 1941 fut de courte durée: en juillet, il fut rattaché au NKVD.

La seconde partition du NKVD se produisit en avril 1943. Cette fois, les organes du contre-espionnage militaire (les Départements spéciaux) ne furent pas rattachés au NKGB mais furent placés sous le commandement direct de Stalin et réorganisés en Direction principale du contre-espionnage (Glavnoe Upravlenie Kontrrazvedki - GUKR), le SMERŠ (Smert’ špionam - Mort aux espions) du commissariat du peuple à la Défense d'URSS. Cependant, le nombre de titulaires du NKGB remodelé en 1943 dépassait de beaucoup celui de 1941 et comptait à ses débuts 67050 agents $^{28}$. Les effectifs postérieurs du NKGB-MGB s'accrurent et, au $1^{\text {er }}$ mai 1946, cet organisme comprenait 115664 personnes. Ce qui ne signifie pas que tous les postes étaient pourvus. Une insuffisance aiguë de cadres s'était fait sentir au cours de la guerre: en 1943, seulement $30 \%$ des postes du NKGB étaient pourvus. Au sortir du conflit, la situation s'était un peu améliorée mais $10,5 \%$ de postes de cadres au MGB étaient encore vacants au $1^{\text {er }}$ mai $1946^{29}$.

Comparativement à la situation d'avant-guerre, on observe un changement dans le niveau de formation des collaborateurs du NKGB-MGB et notamment une tendance à la diminution des agents ayant reçu une formation supérieure, ce qui peut s'expliquer par l'afflux de jeunes cadres qui n'avaient pas pu poursuivre leurs études au cours de la guerre. Ces derniers représentaient la génération qui avait

28. CA FSB, f. 4-os, op. 4, d. 23, 11. 228-230.

29. Ibid. 
néanmoins bénéficié d'une formation secondaire complète (cursus de 10 ans) ou partielle. Il s'ensuivit une tendance générale à la diminution du nombre d'agents dotés seulement d'une formation primaire.

Tableau 10. Niveau de formation des collaborateurs des organes de sécurité d'État ${ }^{30}$

\begin{tabular}{|l|c|c|c|c|}
\hline \multicolumn{1}{|c|}{ Niveau de formation } & $\mathbf{1 . 0 7 . 1 9 3 5}$ & $\mathbf{1 . 0 1 . 1 9 3 8}$ & $\mathbf{1 . 0 1 . 1 9 4 0}$ & $\mathbf{1 . 0 5 . 1 9 4 6}$ \\
\hline supérieur & $1,6 \%$ & $1,4 \%$ & $6,3 \%$ & $3,7 \%$ \\
\hline supérieur inachevé & $\begin{array}{c}\text { données } \\
\text { manquantes }\end{array}$ & $\begin{array}{c}\text { données } \\
\text { manquantes }\end{array}$ & $2,8 \%$ & $2,2 \%$ \\
\hline $\begin{array}{l}\text { secondaire et second. } \\
\text { inachevé }\end{array}$ & $23,8 \%$ & $28,3 \%$ & $36,2 \%$ & $60 \%$ \\
\hline primaire & $74,6 \%$ & $70,3 \%$ & $54,7 \%$ & $34,1 \%$ \\
\hline
\end{tabular}

Cependant, les modifications concernant le niveau de formation du personnel d'encadrement intervenues au cours de la guerre ne sont pas tellement sensibles au sein de la sphère dirigeante de l'appareil central de la Sécurité d'État. Bien qu'à cette échelle, la rotation des cadres ait été considérable durant le conflit (l'arrivée des jeunes cadres est clairement perceptible à travers les changements d'indicateurs concernant le niveau de formation secondaire ou équivalent), il n'y a pas eu au cours de ces années-là de relèvement sérieux du niveau de formation des cadres de l'appareil central.

Tableau 11. Niveau de formation des dirigeants de l'appareil central du NKVD-MGB d'URSS ${ }^{31}$

\begin{tabular}{|l|c|c|}
\hline Niveau de formation & $\mathbf{1 . 0 1 . 1 9 4 1}$ & $\mathbf{1 . 0 5 . 1 9 4 6}$ \\
\hline supérieur & $20,6 \%$ & $29,7 \%$ \\
\hline supérieur inachevé & $5,5 \%$ & $6,3 \%$ \\
\hline secondaire & $38,9 \%$ & $26 \%$ \\
\hline secondaire inachevé & $5,7 \%$ & $13,8 \%$ \\
\hline primaire & $29,3 \%$ & $24,2 \%$ \\
\hline
\end{tabular}

On peut juger des modifications intervenues pendant la période de la guerre d'après la réduction de la proportion des membres formant les couches intermédiaires du parti au regard de l'ensemble des agents du MGB.

30. Le tableau est établi sur la base de matériaux d'archives: GARF, f. 9401, op. 8, d. 40,1. 16 ; d. 50,1.7 ; CA FSB, f. 4-os, op. 4, d. 23,11. 228-230.

31. Le tableau est établi sur la base de matériaux d'archives: GARF, f. 9401, op. 8, d. 64, 1. 6 ; CA FSB, f. 4-os, op. 4, d. 23, 11.228-230. 
Tableau 12. Appartenance au parti des collaborateurs du MGB d'URSS Situation au $1^{\text {er }}$ mai $1946^{32}$

\begin{tabular}{|l|c|c|}
\hline & Tous organes du MGB & $\begin{array}{c}\text { Personnel dirigeant de } \\
\text { l'appareil central du MGB }\end{array}$ \\
\hline membres du VKP(b) & $45 \%$ & $98 \%$ \\
\hline $\begin{array}{l}\text { membres postulants du } \\
\text { VKP(b) }\end{array}$ & $11,7 \%$ & $1,2 \%$ \\
\hline membres du VLKSM* & $23,7 \%$ & - \\
\hline sans appartenance au parti & $19,6 \% * *$ & $0,4 \% * *$ \\
\hline
\end{tabular}

* Vsesojuznyj Leninskij Kommunističeskij Sojuz Molodeži.

** Il s'agit principalement de collaborateurs des directions économiques.

Ici, dans les effectifs de tous les organes du MGB, sont inclus les collaborateurs des secrétariats et des directions économiques (ce qui n'était pas le cas dans les rapports émis avant guerre), c'est pourquoi il serait incorrect de comparer ces chiffres avec ceux fournis plus haut concernant la période 1939-1941.

Après la guerre, les conditions d'embauche dans les organes du MGB ont changé, exigeant un niveau de formation bien précis. Conformément à la décision du Comité central du parti prise le 20 août 1946, une préparation d'enseignement général a été organisée pour les collaborateurs du MGB «sans qu'ils fussent pour autant détachés de leur travail ». Autrement dit, les tchékistes qui n'avaient pas reçu de formation secondaire devaient suivre un enseignement dans des écoles secondaires en cours du soir ou par correspondance. Désormais, il était donc exigé que le postulant à l'embauche dans un poste du MGB eût au moins un formation secondaire. À la fin de l'année 1950, parmi l'ensemble des agents opérationnels du MGB, 75,2 \% étaient dotés d'une formation secondaire et 19,9\% d'une formation supérieure ${ }^{33}$.

L'appartenance au parti des cadres tchékistes est restée un objet de préoccupation particulière. En comparaison avec la situation de 1946, de profonds changements sont intervenus et la couche intermédiaire des agents du parti s'est considérablement accrue. En novembre 1950, 78,5\% des collaborateurs du MGB figuraient comme membres du VKP(b), 6,2\% étaient membres postulants et $12,7 \%$ étaient membres du VLKSM tandis qu'à peine 2,6 \% n'étaient pas inscrits au parti (il s'agissait pour l'essentiel de cadres des sections auxiliaires technico-opérationnelles) ${ }^{34}$.

C'est également à cette époque que s'est clairement affirmée la tendance, déjà notable, à l'homogénéisation nationale des effectifs d'encadrement des services de sécurité. Selon le rapport présenté le 30 novembre 1950 par G. M. Malenkov du MGB au Comité central du parti, la composition nationale des collaborateurs était la suivante:

32. CA FSB, f. 4-os, op. 4, d. 23, 11. 228-230.

33. CA FSB, f. 4-os, op. 8, d. 11,11. 310-341.

34. Ibid. 
Tableau 13. Appartenance nationale des collaborateurs des organes de Sécurité d'État ${ }^{35}$

\begin{tabular}{|l|c|c|c|}
\hline \multicolumn{1}{|c|}{ Nationalité } & $\mathbf{1 . 0 3 . 1 9 3 7}$ & $\mathbf{1 . 0 1 . 1 9 4 1}$ & $\mathbf{3 0 . 1 1 . 1 9 5 0}$ \\
\hline Russes & $65 \%$ & $66 \%$ & $77,1 \%$ \\
\hline Ukrainiens & $11 \%$ & $16 \%$ & $11 \%$ \\
\hline Biélorusses & $4 \%$ & $2,7 \%$ & $1,9 \%$ \\
\hline Géorgiens & $1,2 \%$ & $1,3 \%$ & $1 \%$ \\
\hline Arméniens & $1,8 \%$ & $1,8 \%$ & $1,3 \%$ \\
\hline Azerbaïdjanais & & & $0,4 \%$ \\
\hline Kazakhs & & & $0,8 \%$ \\
\hline Uzbeks & & & $0,3 \%$ \\
\hline Lettons & $1 \%$ & & $0,3 \%$ \\
\hline Lituaniens & & & $0,3 \%$ \\
\hline Estoniens & & & $0,2 \%$ \\
\hline Turkmènes & & & $0,1 \%$ \\
\hline Tadjiks & & & $0,1 \%$ \\
\hline Kirghizes & & & $0,1 \%$ \\
\hline Caréliens et Finnois & & & $0,1 \%$ \\
\hline Moldaves & & & $0,1 \%$ \\
\hline Juifs & & & $1,5 \%$ \\
\hline autres nationalités de l'URSS & & & $0,1 \%$ \\
\hline nationalités « étrangères » & & & \\
\hline
\end{tabular}

Les chiffres concernant la période de travail dans les « organes » tchékistes permettent également de mettre en lumière les années d'afflux massif de cadres dans les organes de sécurité. Le pic intervient ici durant la période allant de 1940 à 1946.

Tableau 14. Répartition des collaborateurs du MGB en fonction de la durée de leur travail au VČK-OGPU-NKVD-MGB ${ }^{36}$

\begin{tabular}{|l|c|}
\hline \multicolumn{1}{|c|}{$\begin{array}{c}\text { Période de travail dans } \\
\text { les organes de la sécurité }\end{array}$} & $\mathbf{3 0 . 1 1 . 1 9 5 0}$ \\
\hline moins d'1 an & $8,1 \%$ \\
\hline 1 à 3 ans & $11,5 \%$ \\
\hline 3 à 10 ans & $50,4 \%$ \\
\hline 10 à 15 ans & $20,8 \%$ \\
\hline 15 à 20 ans & $6,6 \%$ \\
\hline
\end{tabular}

35. Pour rendre les chiffres plus parlants, nous avons inclus les données de 1937 et de 1941. Le tableau est établi sur la base de matériaux d'archives: GARF, f. 9401, op. 8, d. 43, 11. 33-34 ; d. 64,1. 24 ; CA FSB, f. 4-os, op. 8, d. 11,11.310-341. 


\begin{tabular}{|l|l|}
\hline 20 à 25 ans & $1,8 \%$ \\
\hline plus de 25 ans & $0,8 \%$ \\
\hline
\end{tabular}

Au cours de la période 1946-1952, le chiffre des effectifs de la sécurité a continué de croître en raison du transfert du MVD au MGB des troupes de l'intérieur et des gardes-frontières, de la milice et de l'appareil chargé de la surveillance des déportés ainsi que d'autres subdivisions ${ }^{37}$.

Tableau 15. Nombre de collaborateurs des organes du MGB d'URSS Situation au 28 mai $1952^{38}$

\begin{tabular}{|l|c|c|c|c|c|}
\hline & $\begin{array}{c}\text { Organes } \\
\text { du MGB }\end{array}$ & $\begin{array}{c}\text { Troupes } \\
\text { du MGB }\end{array}$ & $\begin{array}{c}\text { Organes du } \\
\text { contre-espion- } \\
\text { nage militaire } \\
\text { du MGB }\end{array}$ & Milice & $\begin{array}{c}\text { Total } \\
\text { au MGB } \\
\text { d'URSS }\end{array}$ \\
\hline $\begin{array}{l}\text { personnel de } \\
\text { commandement } \\
\text { et officiers }\end{array}$ & 120967 & 42046 & 13356 & 95277 & 271646 \\
\hline sergents & 62059 & $\begin{array}{c}\text { données } \\
\text { manquantes }\end{array}$ & 2643 & 102872 & 167574 \\
\hline sans grades & 38822 & 501 & 3892 & 60912 & 104127 \\
\hline total & 221848 & 42547 & 19891 & 259061 & 543347 \\
\hline
\end{tabular}

L'accroissement si brutal de l'appareil de sécurité proprement dit, qui a presque doublé, passant de 115664 à 221848 agents en six ans seulement (de mai 1946 à mai 1952), peut s'expliquer non seulement par l'inflation bureaucratique caractéristique du système soviétique, mais aussi et avant tout par la mise en place des forces de la police secrète chargées de contrôler l'ordre public. Cela témoignait aussi de la crise profonde au sein de la direction du pays qui estimait indispensable d'accroître les effectifs des organes de répression pour assurer la stabilité du régime.

(Traduit du russe par Françoise Cordes-Baudrillard et Catherine Klein-Gousseff)

Memorial-Moscou

Malyj Karetnyj per., 12

$103051 \mathrm{Moscou}$

36. CA FSB, f. 4-os, op. 8, d. 11,11.310-341.

37. Pour plus de détails, cf. A. Kokurin, N. Petrov, « MGB: struktura, funkcii, kadry » (MGB: structures, fonctions, encadrement), Svobodnaja mysl', 11, 1997, p. 112.

38. CA FSB, f. 4-os, op. 10, d. 73,1.177. 